\title{
Towards a Micro-Macro Model of Hot Tearing
}

\author{
M. Rappaz ${ }^{1, a}$, J.-M. Drezet ${ }^{1,2, b}$, V. Mathier ${ }^{1, c}$ and S. Vernède ${ }^{1,3, d}$ \\ ${ }^{1}$ Computational Materials Laboratory, School of Engineering, \\ Ecole Polytechnique Fédérale de Lausanne, Station 12, CH-1015 Lausanne, Switzerland \\ ${ }^{2}$ Calcom-ESI SA, PSE-Ecublens, CH-1015 Lausanne, Switzerland \\ ${ }^{3}$ Alcan-CRV, F-38341 Voreppe, France \\ amichel.rappaz@epfl.ch, bjean-marie.drezet@epfl.ch, \\ cvincent.mathier@epfl.ch, ${ }^{d}$ stephane.vernede@epfl.ch
}

Keywords: solidification, hot tearing, feeding, coalescence.

\begin{abstract}
Modelling of hot tearing must encompass several aspects: solidification, percolation of grains, stress/strain development, liquid feeding. The present contribution reviews recent progress made in this domain, both at the macro- and microscopic levels. At the macroscopic level, average two-phase approaches based on the coupling of a porous medium model for the solid skeleton and liquid feeding (Darcy model) will be presented briefly. Such models provide a clear enhancement of the RDG model[1], especially from a rheology point of view. At the microscopic level, a new granular approach has been undertaken recently. Considering in a first step the solidification and coalescence of equiaxed grains, it has been extended recently to the calculation of feeding using a Kirchhoff-Poiseuille (KPL) model. Percolation of grains and localisation of feeding could be clearly evidenced in this way.
\end{abstract}

\section{Introduction}

Hot tearing together with microporosity have always been recognized as major defects in castings of aluminum alloys, the first one being typical of semi-continuous casting whereas the second one is dominant in shape castings. As explained in the textbook of Campbell[2], these two defects are interconnected, as both result from a lack of feeding and nucleation of a pore/void in the remaining liquid. However, if porosity is associated with solidification shrinkage and can occur within the grains or at grain boundaries, hot tearing is clearly linked with tensile stresses in the solid and is confined to grain boundaries.

The purpose of this contribution is not to review all the hot tearing models since a review has been published by Eskin et al[3] in 2004. It is rather to present recent developments made both at the macroscopic and microscopic levels, pointing out the difficulties in connecting these two scales. Macroscopic approaches, which are presented in the next section, are based nowadays on two-phase approaches[4-6]: they are the natural extension of the so-called RDG criterion[1], but using a more rigorous rheological approach for the compressible mushy solid phase. The main advantage of such methods is that they can take into account the whole scale of a casting, but their weakness is that averages can hardly account for the localization of strains and feeding at grain boundaries. On the microscopic scale, coalescence and percolation of grains can be accounted for at the scale of a small volume element of the mushy zone (typically a few cubic centimeters) using granular approaches[79]. Although such techniques cannot yet consider a whole solidification process, they provide a detailed and interesting view of the phenomena occurring during hot tearing, in particular localization of strains and feeding, gradual transition from a continuous liquid film network to a fully coherent solid, etc. These methods are briefly presented in the next section. Finally, results obtained with both macro- and micro-approaches are presented and discussed before some conclusion. 


\section{Macroscopic two-phase semi-coupled approach of hot tearing}

In the RDG criterion[1], a strain rate perpendicular to the thermal gradient was applied to the solid phase, regardless whether it corresponds to columnar or equiaxed dendrites (see Fig. 1). The component of the strains parallel to the thermal gradient was neglected on the basis that it is not a component susceptible of inducing hot tearing. Defining then the average density of the solid-liquid mixture as $\rho=\rho_{s} g_{s}+\rho_{\ell} g_{\ell}$, where $g_{v}$ and $\rho_{v}$ are the volume fraction and the density of phase $v$ $(v=s, \ell)$, and using Darcy's equation describing the interdendritic flow in a mushy zone, the following expression was derived for unsteady conditions:

\section{Erreur $!=0$}

$\beta=\left(\rho_{\mathrm{s}} / \rho_{\ell}-1\right)$ is the solidification shrinkage, $\varepsilon,{ }_{\mathrm{s}_{\perp_{1}}}$ and $\varepsilon,{ }_{\mathrm{s}_{2}}$ are the two components of the strain rate of the solid perpendicular to the thermal gradient (see Fig. 1), $\mathrm{K}$ is the permeability of the mush, $\mu$ is the viscosity and $p_{\ell}$ the local pressure in the interdendritic liquid, and $\mathbf{g}$ is the gravity vector. Please note that $\rho_{\mathrm{s}}$ and $\rho_{\ell}$ have been assumed constant, while $g_{\mathrm{s}}$ is constant in the directions perpendicular to the thermal gradient. This expression is fairly general and can be interpreted as follows: solidification shrinkage $\left(1^{\text {st }}\right.$ term) and/or deformation of the solid ( $2^{\text {nd }}$ term) have to be compensated by liquid flow ( $3^{\text {rd }}$ term) if pores or hot tears are to be avoided. In the case a third phase (pores or hot tears) is considered, the right hand term of Eq. 1 is simply replaced by $-\partial \mathrm{g}_{\mathrm{p}} / \partial \mathrm{t}$, where $g_{p}$ is the fraction of pores (or hot tears).

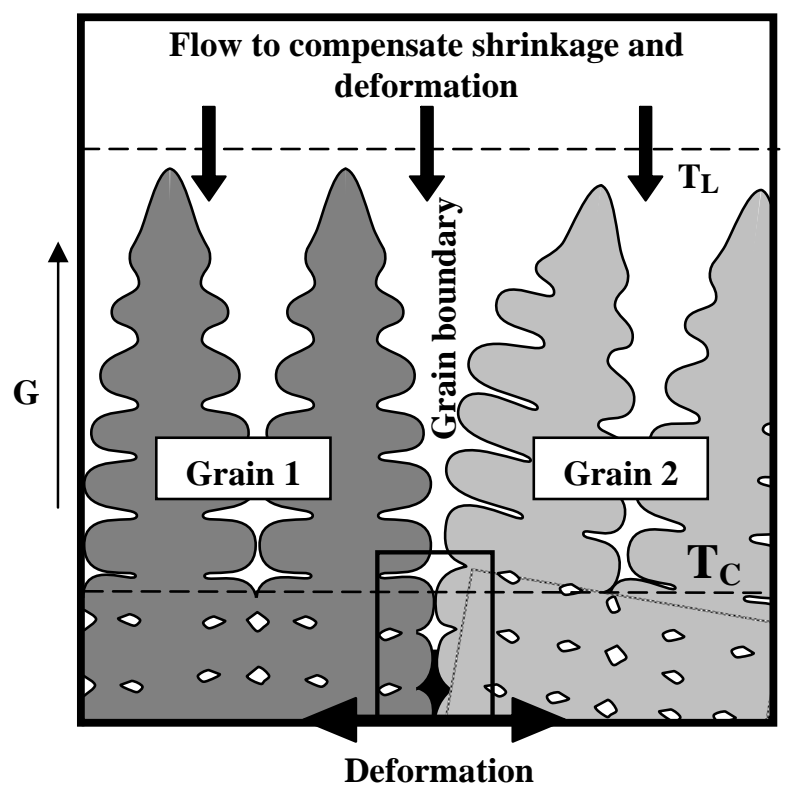

Figure 1: Schematics of hot tearing formation and of the two-phase problem considered in the RDG approach.[1].
Under steady directional solidification conditions at a velocity $\mathrm{v}_{\mathrm{T}}$, the maximum pressure drop, $\Delta \mathrm{p}_{\max }$, across the mushy zone and associated with deformation and solidification shrinkage is given by:

\section{Erreur! (2)}

where $\mathrm{E}$ is the cumulated strain rate, i.e., Erreur!.

These two integrals can be transformed into integrals over temperature, thus introducing a "competition" between strain rate and thermal gradient, $\mathrm{G}$, for the first contribution, and the standard $\mathrm{v}_{\mathrm{T}} / \mathrm{G}$ ratio for the shrinkage term (i.e., already derived by Niyama in his porosity criterion[10]).

The RDG criterion simply states that a hot tear will form if the local pressure in the liquid, i.e., the metallostatic pressure minus the pressure drop, falls below a given cavitation pressure. It offers two main advantages: (1)

Fixing $\Delta \mathrm{p}_{\max }$ to a preset value (e.g., $2-5 \mathrm{kPa}$ ), Eq. (2) allows to set up a hot cracking sensitivity of an alloy by calculating the corresponding $\mathrm{E}_{\max }$; (2) Using a simple rheology for the solid phase, e.g., Norton law, the actual plastic strains can be computed everywhere in a casting. Although these strains will satisfy the incompressibility condition, the two contributions perpendicular to the gradient will not and the actual pressure in the liquid can be calculated using Eq. (1). This criterion has nevertheless a few limitations: (1) Using only the perpendicular component of the plastic strains is not strictly valid, the longitudinal component also inducing some suction (or expulsion) of the liquid; (2) The upper bound of the integrals of Eq. (2) is ill-defined. As $g_{s}$ tends towards unity, the 
permeability goes to zero and the calculation diverges. In practical situations, this bound is set up to a value of $g_{s}$ at which the solid is considered as coherent, i.e., the liquid remains only as liquid pockets (no continuous liquid films). However, this coherency value is certainly not the same within a grain or at a grain boundary (see below the aspects of coalescence[11], Fig. 1); (3) In relation to grain boundaries, the method does not consider any localization of the strains and feeding.

In order to overcome the first limitation, M'Hamdi et al[4,5] introduced a more rigorous formalism for the deformation of the solid. While the mass conservation equation (1) remains the same, these authors coupled this equation with the momentum equation for the solid phase. As the solid is in contact with the liquid phase, an effective stress acting on the solid, $\sigma_{\mathrm{s}}$, was introduced. Being equal to $\left(\sigma+p_{\ell} \mathbf{I}\right)$, where $\sigma$ is the total stress and $\mathbf{I}$ is the identity tensor, the equilibrium condition becomes:

$\operatorname{div} \sigma_{\mathrm{s}}+\rho \mathbf{g}=\operatorname{grad} \mathrm{p}_{\ell}$

M'Hamdi et al used a complex rheological behaviour for the mushy solid derived by Ludwig et al $[12,13]$, in which the plastic strain rate is a function not only of the equivalent von Mises stress but also of the pressure in the solid (compressibility of the mushy solid) and of a cohesion parameter. They solved this coupled problem (Eqs (1) and (3)) using a specific Finite Element code in which the pressure in the liquid and the displacements (strains) of the solid are the unknowns. However, considering that pressure drops across the mushy zone encountered in many casting processes are of the order of a few $\mathrm{kPa}$ while stresses in the coherent mush are of the order of a few $\mathrm{MPa}$, it seems reasonable to neglect the effect of liquid pressure on the solid skeleton mechanical behaviour. In this case, grad $\mathrm{p}_{\ell}$ can be neglected and the equation of the solid becomes independent of the liquid problem. The advantage of such a decoupling, developed by Mathier et al[6], is that the mechanical problem (Eq. (3) with $\mathrm{p}_{\ell}=0$ ) can be solved independently of the fluid flow problem (Eq. (1)). From a thermal field calculation giving the temperature $\mathrm{T}$ and $\mathrm{g}_{\mathrm{s}}$, the strains are computed using the rheology of a porous (instead of mushy) solid as derived by Ludwig et al. This was done using the Abaqus ${ }^{\circledR}$ code and appropriate user functions for the rheological law. As the porous solid is compressible, the divergence of $\mathbf{v}_{\mathrm{s}}$, which is non-zero, can be computed as the trace of the plastic strain rate. This contribution is then used as the second term of Eq. (1) for the computation of the pressure in the liquid and if needed the fraction of porosity, $\mathrm{g}_{\mathrm{p}}$. This was achieved using the approach developed by Pequet et al[14] and the software CalcoSOFT ${ }^{\circledR}$.

\section{Granular model of hot tearing}

Although an appropriate two-phase approach alleviates the first drawback of the RDG criterion mentioned in the previous section, it does not remove the others. In particular, average methods are unable to account for the localization of hot tears at grain boundaries. This localization is essentially due to the fact that liquid films remain to lower temperature as compared to those located in between dendrites of the same grains (Fig. 1). In other words, the formation of a coherent solid network by coalescence or bridging of dendrites arms occurs earlier within the grains as compared with grain boundaries. Rappaz et al[11] have introduced for that purpose a coalescence or bridging undecooling which, for a pure metal, is given by:

\section{Erreur!}

where $\gamma_{\mathrm{gb}}$ is the grain boundary energy, $\gamma_{\mathrm{s} \ell}$ is the solid-liquid interfacial energy, $\Delta \mathrm{s}_{\mathrm{f}}$ is the volumetric entropy of fusion and $\delta$ is the thickness of the diffuse interfaces. For an alloy, coalescence is reached when a coalescence line (or surface) parallel to the liquidus, but $\Delta \mathrm{T}_{\mathrm{b}}$ below, is reached. Within a grain, there is no grain boundary energy and interfaces become attractive as 
soon as they get within interaction distance, i.e., distance $\delta$. At "repulsive" grain boundaries, $\gamma_{\mathrm{gb}}>$ $2 \gamma_{\mathrm{s} \ell}$, bridging is reached at some $\Delta \mathrm{T}_{\mathrm{b}}>0$.

This concept of bridging undercooling has been tested by phase field[11], by molecular dynamics[15] and by experiment[16]. This experiment is worth mentioning: two crystals of a nickel-base superalloy were laser welded together under well defined conditions with increasing misorientations. At small misorientation (typically less than $15 \mathrm{deg}$.), no hot crack formed along the weld centreline, whereas at larger values, a crack was initiated under the same conditions, thus showing the influence of the grain boundary energy. The simulations done by molecular dynamics or by phase field are interesting but correspond to very small regions near the incoming interfaces, typically a few tens of nanometers or micrometers, respectively. In practical situations, hot tears are indeed located at grain boundaries, but the configuration of these boundaries associated with nucleation and growth of grains is essential.

For this reason, a simplified approach of coalescence for a large population of equiaxed grains was undertaken first by Mathier et al[7] and then by Vernède et al[8,9]. In this granular approach, a random set of nucleation centres with random orientations is first generated in a given volume. Considering that the grains nucleate at the same time and that the temperature difference across each grain is small with respect to the growth undercooling, the grain boundaries correspond to the Voronoï tessellation of the nucleation centres (Fig. 2a), i.e., the grain boundary between grains I and $\mathrm{K}$ is the median line. Assuming globular grains, the smooth solid-liquid interfaces is first approximated by linear segment in each triangle linking a nucleation centre (open circle) and two vortices of the tessellation (open squares) (Fig. 2b). Solidification is then calculated within each triangle using a microsegregation model[7-9]. Therefore, at any time, the remaining width of the liquid channel in between two grains is known providing the thermal field is known. When the two solid-liquid interfaces get within interaction distances, coalescence is accounted for, using Eq. (4) and a Read-Schockley grain boundary energy model.

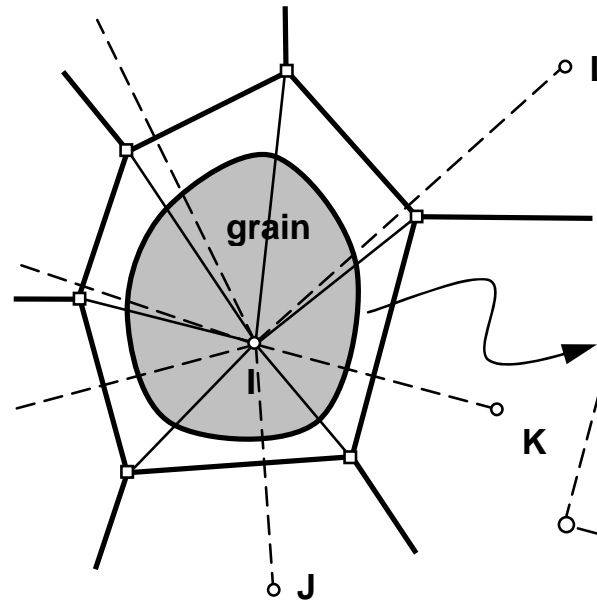

(a)

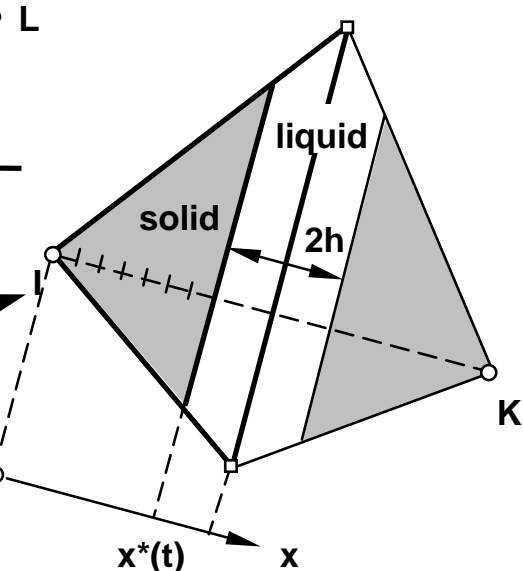

(b)

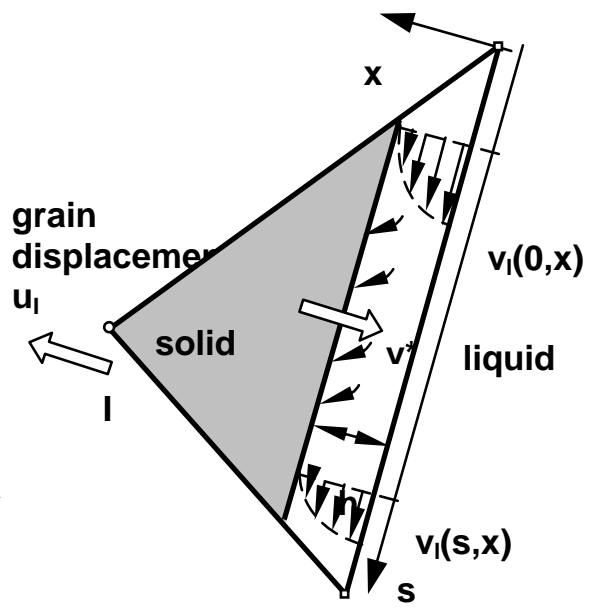

(c)

Figure 2: Schematics of the granular model used for the simulation of solidification and feeding in a network of equiaxed globular grains: Voronoï tessellation (a), microsegregation model (b), feeding KPL model (c).

The next step undertaken is to calculate feeding within the network of liquid films. For that purpose, a Poiseuille flow was first assumed within the channels (Fig. 2c)[8,9]. This flow is not constant along a given channel as it has to feed solidification shrinkage and the relative movement of grains, i.e., the flow has some Losses along each channel. Finally, at each vortex of the Voronoï 
tessellation, the sum of the (signed) incoming flows must be zero, i.e., Kirchhoff's law. This KPL model is similar to a network of electrical resistances which would not be well insulated from the circuit board (losses). Within each channel (resistance), the flow is governed by the following equation:

\section{Erreur!}

where $p_{\ell}$ is the pressure in the liquid film, $h$ is the half thickness of the remaining liquid film, $s$ is the coordinate along the film and $\mathrm{v}^{*}$ is the speed of the solid-liquid interface. Please note that a cubic dependence of the liquid film thickness is found for the pressure drop in the liquid films and that this equation is of the same nature as Eq. (1), i.e., a second-order differential equation for the pressure field with a sink term given by solidification shrinkage. Provided appropriate boundary conditions are set, Eq. (5) can be solved using a Finite Element method with linear functions along each liquid channel. When the grains move with respect to each other, this equation can be modified in a way similar to Eq. (1), i.e., introducing the normal relative velocities of the nodes, $\left(\mathbf{v}_{\mathrm{I}} \cdot \mathbf{n}_{\mathrm{IK}}+\mathbf{v}_{\mathrm{K}} \cdot \mathbf{n}_{\mathrm{KI}}\right)$, where $\mathbf{n}_{\mathrm{IK}} / \mathbf{n}_{\mathrm{KI}}$ are the normals to the interface issued from nodes $\mathrm{I} / \mathrm{K}$, respectively. The displacement of the nodes can be calculated using a mechanical model which is under development.

\section{Results and discussion}

The result shown in Fig. 3 corresponds to a two-phase macroscopic calculation for the start-up phase of an axisymmetric DC cast AA5182 alloy. The diameter of the billet is $247 \mathrm{~mm}$ and the casting speed was ramped from $90 \mathrm{~mm} / \mathrm{min}$ to $150 \mathrm{~mm} / \mathrm{min}$ in $500 \mathrm{~s}$ producing $1 \mathrm{~m}$ of casting. The mechanical properties of the AA5182 alloy are available both in the mushy state[12.13] and for the solid alloy at various temperatures[17]. Once the strains and strain rates were calculated with Abaqus, the volumetric plastic strain rate contribution, $\operatorname{div}\left(\mathbf{v}_{\mathrm{s}}\right)$, was implemented into the porosity model of Pequet et al[14], but assuming that no hydrogen was dissolved initially in the melt (i.e., no pore formed). Fig. 3a shows the pressure drop calculated after $500 \mathrm{~s}$ of casting, but neglecting plastic deformation of the solid. In the liquid pool, one can notice a small increase of the pressure due to the metallostatic head. The pressure drop in the mushy zone associated with solidification shrinkage only is most pronounced at the centre of the billet: this can be expected since the thermal gradient is the lowest (large extent of the mushy zone) and the velocity of the isotherms is the largest (large liquid suction). Fig. $3 \mathrm{~b}$ shows the pressure drop increase due to the volumetric plastic strains: as the mushy solid is under dilatant conditions near the center of the billet, an additional depression of about $4 \mathrm{kPa}$ is calculated. This is a fairly small contribution as compared with the $27 \mathrm{kPa}$ associated with solidification shrinkage. The cumulated volumetric strain, i.e., the integral over the solidification interval of $\operatorname{div}\left(\mathbf{v}_{\mathrm{s}}\right)$, is indicated in Fig. $3 \mathrm{c}$ : it is only $0.74 \%$ while solidification shrinkage amounts to $6.5 \%$, thus explaining the relative importance to the pressure drop contribution. Finally, Fig. $3 d$ shows the strain rate calculated at $t=500$ s. Similar calculations have been performed with some initial amount of dissolved hydrogen.[6] In this case, some porosity/hot tears is present in the casting, the formation of which relaxing slightly the pressure drop. 


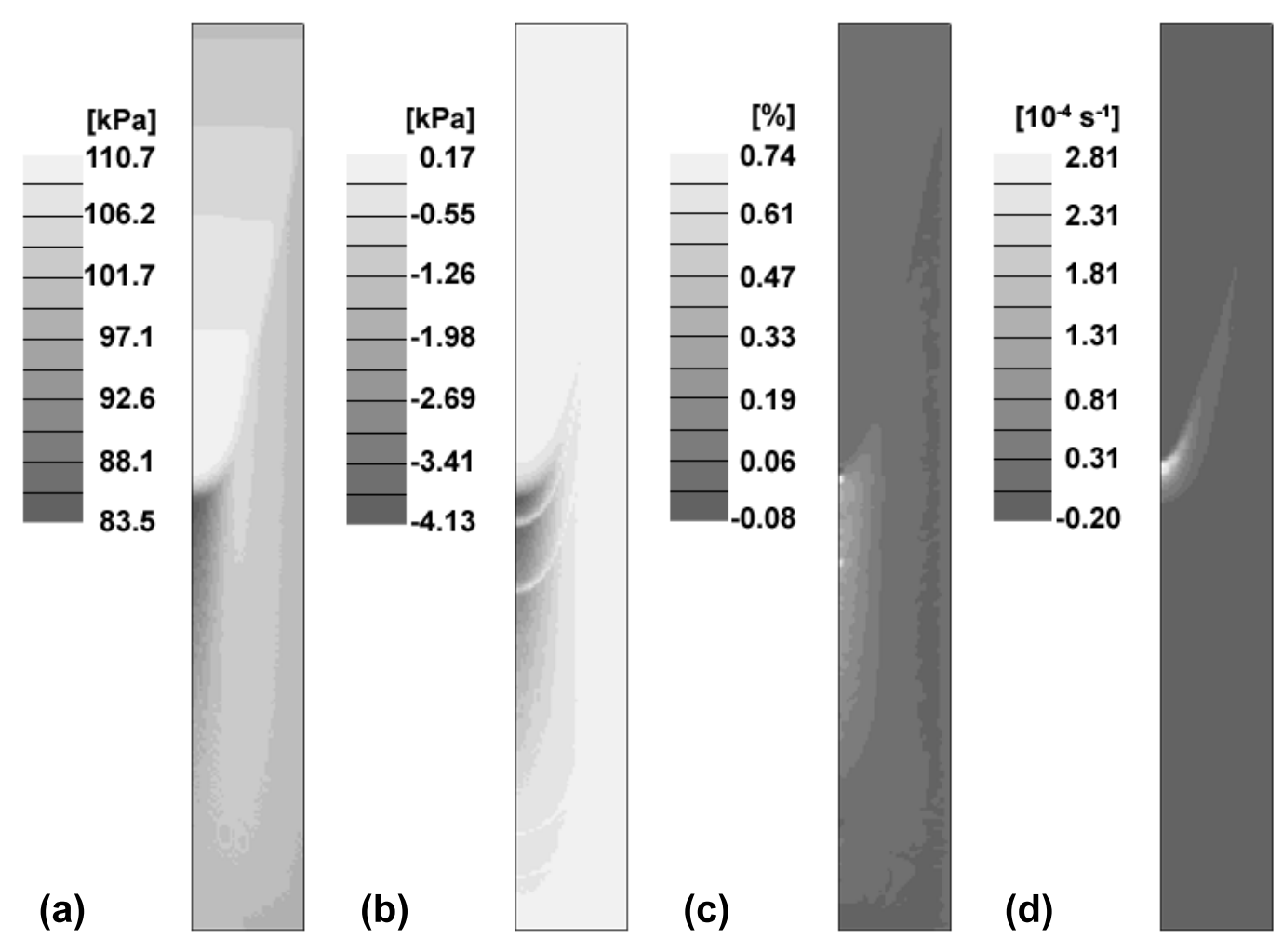

Figure 3: Results at 500s in a DC cast AA5182 alloy showing the effect of volumetric strain (a) liquid pressure neglecting the solid deformation contribution (b) pressure difference due to deformation only (c) cumulated volumetric strain $(\mathrm{d})$ volumetric strain rate at that time.

The granular model has been applied to the directional solidification of an $\mathrm{Al}-1 \% \mathrm{Cu}$ alloy (Fig. 4). The thermal gradient was $60 \mathrm{~K} / \mathrm{cm}$ and the cooling rate $-1 \mathrm{~K} / \mathrm{s}$ (i.e., velocity of the isotherms equal to $170 \mu \mathrm{m} / \mathrm{s}$ ). The average grain density was set to $10^{8} \mathrm{~m}^{-2}$, i.e., average grain size of $100 \mu \mathrm{m}$, and the computation domain which spans across the whole mushy zone contains 14'000 grains. The central figure in Fig. 4 shows the grains with various grey levels, grains in solid contact (clusters) being represented with the same grey level while the liquid films are in black. The small figures on the left are magnifications of 4 typical regions of the mushy zone which are discussed hereafter. On the right of Fig. 4, the evolution of the volume fraction of solid as calculated in horizontal sections of the grain structure is represented together with the imposed temperature profile.

In region (a), typically for $0<\mathrm{g}_{\mathrm{s}}<0.89$, most the grains are isolated and surrounded by liquid films. For $0.89<\mathrm{g}_{\mathrm{s}}<0.97$ (region (b)), clusters of a few grains are formed but the liquid films remain continuous and interconnected. In region (c) characterised by $0.97<\mathrm{g}_{\mathrm{s}}<0.99$, larger clusters are visible, with a few isolated liquid films remaining inside. Finally, in region (d) $(0.99<$ $\mathrm{g}_{\mathrm{s}}<1$ ), the solid network is continuous and liquid only remains as isolated regions. As can be seen, this granular model is able to predict the gradual transition from a continuous intergranular film network to a continuous fully coherent solid. It should be emphasised that cluster formation is directly induced by the stochastic nature of the nucleation centre locations, a feature that has not been considered in past simulation works related to hot tearing, and to a lower extent by coalescence. Further analysis of the transition regions is given in [9]. Finally, the results shown here have been obtained with a model in which the polygonal grains were slightly rounded at the corners. This was done considering the curvature undercooling and a solute balance, the details of which will be given elsewhere. This "rounding" procedure does not change the topological 
observations, but slightly decreases the volume fraction of solid at which the topological transitions occur.

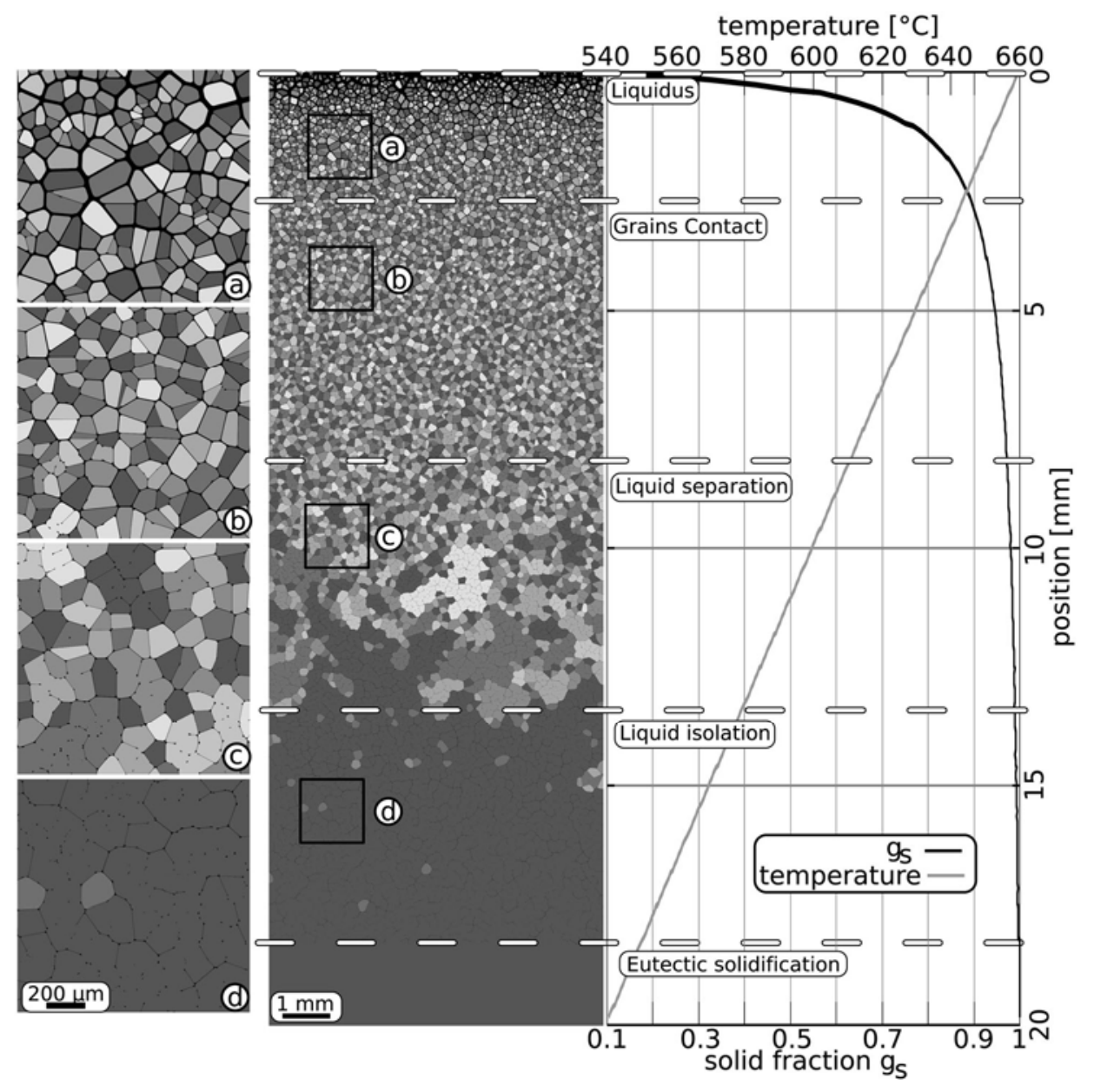

Figure 4: Calculated mushy zone for an Al-1wt\%Cu alloy cooled down at $-1 \mathrm{~K} / \mathrm{s}$ in a gradient of $6000 \mathrm{~K} / \mathrm{m}$. Grains in solid contact are shaded with the same grey level.

Using the grain structure calculated with the granular model, KPL calculations can be performed to evaluate the average feeding and its localisation. In [8,9], it has been shown that the overall feeding behaviour predicted with the KPL approach follows the Carman-Kozeny relationship. However, at high volume fraction of solid, feeding is highly localised and starts to deviate from this law as many liquid channels are no longer connected to the feeding network but contribute to $g_{\ell}$. Figure 5 shows a portion of the mushy zone of an $\mathrm{Al}-1 \mathrm{wt} \% \mathrm{Cu}$ at 0.98 solid fraction (the temperature in this case is assumed uniformed). The liquid flow on the right side of the domain is assumed to be zero, while the pressure is imposed to zero on the left side. The horizontal boundaries are closed. In this case, fluid flow is only induced by solidification shrinkage: this situation thus corresponds to feeding condition deep in the mush. The grey scale in Fig. 5 is an indication of the (negative) pressure in the liquid phase. Although the pressure is defined only in the liquid phase, the grey scale is also represented in the grains for visibility. White areas correspond to liquid channels which are not connected any more to the main liquid pocket on the left and for which the pressure is no longer calculated. The width of the liquid channels has been magnified by a factor proportional to the local flow, i.e., thick/thin lines correspond to large/small flows. 


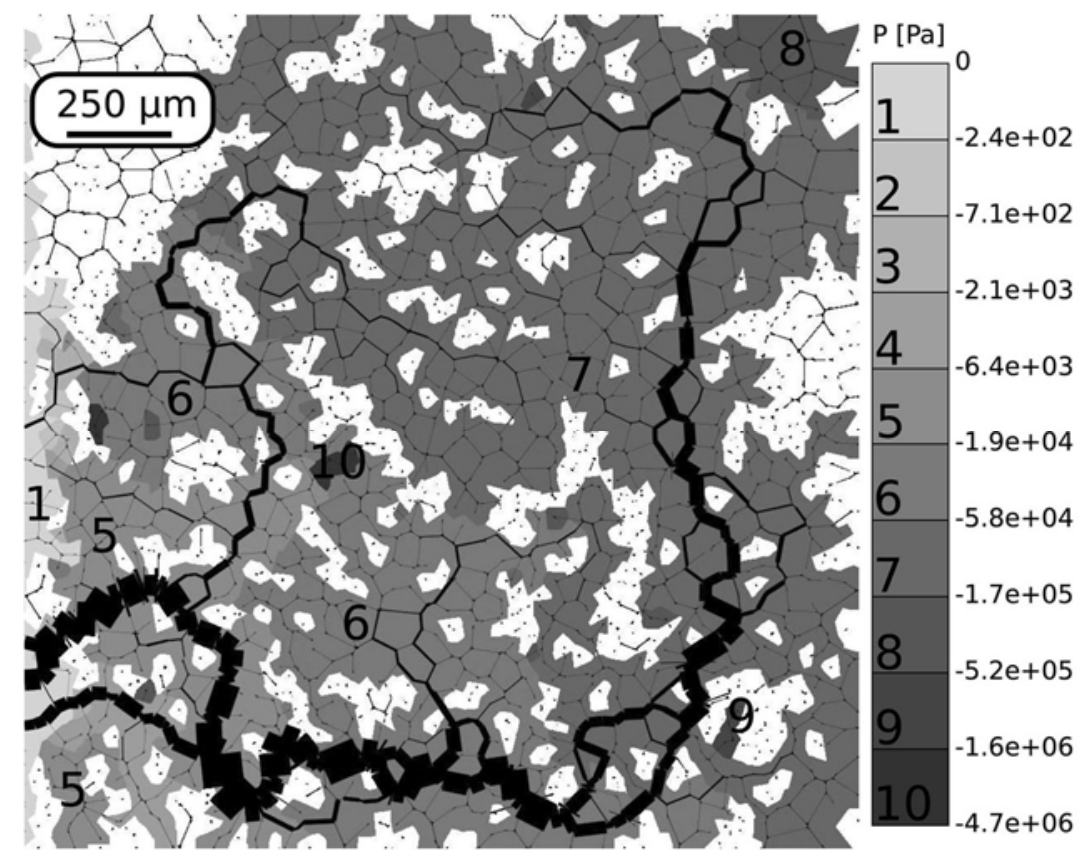

Figure 5: Pressure drop and fluid flow induced by solidification shrinkage in a 2 $\times 2 \mathrm{~mm}^{2}$ mushy zone element. In this sample, $\mathrm{T}=590{ }^{\circ} \mathrm{C}$ and $\mathrm{g}_{\mathrm{s}}=0.984$. The width of each channel is magnified proportionally to the local flow, the pressure scale is indicated on the right.

At this high volume fraction of solid, it appears that there is mainly one path of connected liquid channels that feed the entire mush. Along this feeding path, the pressure goes from 0 (region 1) to $170 \mathrm{kPa}$ (region 7). It is clear that under such large depression, porosity should form in order to relax the pressure drop. Besides unfed isolated liquid channels, a few channels still connected to the liquid source exhibit very large pressure drops (typically MPa, regions 9 and 10). Such large values are on the order of magnitude of the plastic stress of the solid phase at high temperature. When displacement and deformation of the solid grains will be implemented in this granular model, such occurrence should disappear.

\section{Conclusion}

Hot tearing is a complex defect that involves many phenomena, in particular thermal and solidification aspects, stress-strain in an increasingly coherent solid, feeding in a gradually disappearing liquid film network. The RDG criterion provided the first two-phase approach, which was then further improved using a more rigorous formalism and the complex rheology of porous media. Nevertheless, these approaches are still based on averages and do not consider any localization of strains and feeding at grain boundaries. Granular models, while still limited to small portions of a solidification process, have certainly the potential to answer some of these questions, once mechanical aspects will be fully built in and the model will be extended to 3 dimensions. The numerical simplicity of such approaches makes it feasible from a CPU time point of view.

\section{Acknowledgements}

The authors would like to thank Ph. Jarry and B. Commet from the Alcan-Pechiney, Research Center of Voreppe (CRV), France, for fruitful discussions. The authors would like to thank also the Commission for Innovation and Technology, CTI, Bern (grant 6167.1 KTS), and the industries Alcan (CH), Alcan (FR), Calcom-ESI (CH), HydroAluminium (DE), General Motors (USA) and Union Minière $(\mathrm{BE})$ for their financial support. 


\section{References}

[1] M. Rappaz, J.-M. Drezet and M. Gremaud: Met. Mat. Trans. 30A (1999), 449.

[2] J. Campbell: Castings (Elsevier, 2003).

[3] D.G. Eskin, Suyitno and L. Katgerman: Progr. Materials Science 49 (2004) 629.

[4] M. M'Hamdi, A. Mo, and C.L. Martin: Met. Mat. Trans. 33A (2002) 2081.

[5] M. M'Hamdi, H.G. Fjaer, A. Mo, D. Mortensen and S. Benum: A New Two-phase Thermomechanical Model and its Application to the Study of Hot Tearing Formation During the Start-up Phase of DC Cast Ingots. in TMS 2004.

[6] V. Mathier, J.-M. Drezet and M. Rappaz: Two-Phase Modeling of Hot Tearing in Aluminum Alloys Using a Semi-Coupled Method, in Modeling of Casting, Welding and Advanced Solidification Processes, Eds. Ch. Gandin et al (TMS Publ., Warrendale, USA, 2006), to appear.

[7] V. Mathier, M. Rappaz and A. Jacot: Mod. Simul. Mater. Sc. Engng 12 (2004) 479.

[8] S. Vernède and M. Rappaz: Transition of the Mushy Zone from Continuous Liquid Films to a Coherent Solid, Phil. Mag. (2006) to appear.

[9] S. Vernède and M. Rappaz: A Granular Model of Mushy Zones: Formation of a Coherent Solid and Localization of Feeding, to be published.

[11] E. Niyama, T. Uchida, M. Morikawa and S. Saito: AFS Int. Cast Metals J. (Sept.1982) 52.

[12] O. Ludwig, J.-M. Drezet, C.L. Martin, and M. Suéry: Met. Mat. Trans. 36A (2005) 1525.

[13] O. Ludwig, J.-M. Drezet, P. Ménésès, C.-L. Martin, and M. Suéry: Mater. Sci. and Engng A, 413-414 (2005) 174.

[14] C. Péquet, M. Gremaud and M. Rappaz: Met. Mat. Trans. 33A (2002) 2095.

[15] A. Karma, private communication.

[16] N. Wang, S. Mokadem, M. Rappaz and W. Kurz: Acta Mater. 52 (2004) 3173.

[17] W.M. Van Haaften, W.H. Kool and L. Katgerman: Mat. Sc. Engng A336 (2002) 1. 